\title{
Anemoside A3 Enhances Cognition through the Regulation of Synaptic Function and Neuroprotection
}

\author{
Fanny CF Ip ${ }^{1,2,3,4,7}$, Wing-Yu Fu ${ }^{1,2,3,7}$, Elaine YL Cheng ${ }^{1,2,3}$, Estella PS Tong ${ }^{1,2,3}$, Ka-Chun Lok ${ }^{1,2,3}$, Yan Liang ${ }^{1,2,3}$, \\ Wen-Cai Ye $e^{*, 4,5,6,8}$ and Nancy $Y$ Ip ${ }^{*, 1,2,3,4,8}$ \\ 'Division of Life Science, The Hong Kong University of Science and Technology, Hong Kong, China; ${ }^{2}$ Molecular Neuroscience Center, The Hong \\ Kong University of Science and Technology, Hong Kong, China; ${ }^{3}$ State Key Laboratory of Molecular Neuroscience, The Hong Kong University of \\ Science and Technology, Hong Kong, China; ${ }^{4}$ HKUST-Jinan Joint Laboratory of Innovative Drug Discovery, Jinan University, Guangzhou, China; \\ ${ }^{5}$ Institute of Traditional Chinese Medicine and Natural Products, College of Pharmacy, Jinan University, Guangzhou, China; ${ }^{6}$ Guangdong Province \\ Key Laboratory of Pharmacodynamic Constituents of Traditional Chinese Medicine and New Drugs Research, Jinan University, Guangzhou, China
}

\begin{abstract}
Compounds that have the ability to both strengthen synaptic function and facilitate neuroprotection are valuable cognitive enhancers that may improve health and quality of life, as well as retard age-related cognitive deterioration. Medicinal plants are an abundant source of potential cognitive enhancers. Here we report that anemoside A3 (AA3) isolated from Pulsatilla chinensis modulates synaptic connectivity in circuits central to memory enhancement. AA3 specifically modulates the function of AMPA-type glutamate receptors (AMPARs) by increasing serine phosphorylation within the GluAI subunit, which is a modification required for the trafficking of GluA I-containing AMPARs to synapses. Furthermore, AA3 administration activates several synaptic signaling molecules and increases protein expressions of the neurotrophin brain-derived neurotrophic factor and monoamine neurotransmitters in the mouse hippocampus. In addition to acting through AMPARs, AA3 also acts as a non-competitive NMDA receptor (NMDAR) modulator with a neuroprotective capacity against ischemic brain injury and overexcitation in rats. These findings collectively suggest that AA3 possesses a unique ability to modulate the functions of both AMPARs and NMDARs. Concordantly, behavioral studies indicate that AA3 not only facilitates hippocampal long-term potentiation but also enhances spatial reference memory formation in mice. These multifaceted roles suggest that AA3 is an attractive candidate for further development as a cognitive enhancer capable of alleviating memory dysfunctions associated with aging and neurodegenerative diseases.
\end{abstract}

Neuropsychopharmacology (2015) 40, 1877-1887; doi:I0.1038/npp.2015.37; published online 4 March 2015

\section{INTRODUCTION}

Dementia is a progressive decline of cognitive functions such as learning and memory. It is a crucial problem in the elderly population and a hallmark of age-related afflictions such as Alzheimer's disease. Age-related memory loss is thought to be associated with a progressive weakening of synaptic function and loss of excitatory synapses (Palop and Mucke, 2010; Sheng et al, 2012). The enhancement or maintenance of normal synaptic structures and function in healthy individuals improves cognitive performance in daily life and may also delay the initiation or slow the progression of disease-related cognitive impairment.

*Correspondence: Professor NY Ip, Division of Life Science, The Hong Kong University of Science and Technology, Clear Water Bay, Hong Kong 000, China, Tel: +852 2358 7269, Fax: +852 2358 |464, E-mail: BOIP@UST.HK or Professor W-C Ye, Institute of Traditional Chinese Medicine and Natural Products, College of Pharmacy, Jinan University, Guangzhou, China, Tel: +8620 8522 0936, Fax: 8620-85221559, E-mail: CHYWC@YAHOO.COM.CN

${ }^{7}$ These authors contributed equally to this work.

${ }^{8}$ These authors are the co-corresponding authors.

Received 23 September 2014; revised 16 January 2015; accepted 21 January 2015; accepted article preview online 4 February 2015
Several strategies for cognitive enhancement have been proposed, such as the modulation of synaptic protein synthesis, facilitation of synaptic function by modifying synaptic transmission and connectivity, augmentation of adult neurogenesis, and prevention of neuronal death to protect deteriorating neural networks. Glutamate transmission, which is propagated by the two major ionotropic glutamate receptor subtypes, the AMPA-type glutamate receptors (AMPARs) and NMDA receptors (NMDARs), is critical for regulating synaptic strength in the hippocampus. These receptors are highly expressed at excitatory synapses in the hippocampus; they mediate basal excitatory synaptic transmission and regulate synaptic plasticity, which are the fundamental molecular basis of learning and memory (Anggono and Huganir, 2012; Paoletti et al, 2013; Riedel et al, 2003). Although AMPARs are responsible for mediating basal synaptic transmission, NMDAR activation has essential roles in modulating activity-dependent synaptic plasticity via calcium-mediated signaling pathways and protein synthesis (Rebola et al, 2010). Augmentation of ionotropic glutamate receptor function enhances neurotransmission, which is believed to affect memory and cognitive performance. This can be achieved by regulating the receptors' subcellular trafficking, duration of 
channel opening, or associated scaffold proteins and downstream signalers (Yokoi et al, 2012; Kumar and Mayer, 2013).

Although ionotropic glutamate receptor activation is essential for synaptic strength and cognition, their hyperactivation triggers excessive influx of calcium ions, resulting in synaptic dysfunction and neuronal damage (Chang et al, 2012; Kawahara et al, 2004; Paoletti et al, 2013). NMDAR overactivation is believed to be a major contributing factor in the development of neurological disorders and neurodegenerative diseases such as depression, ischemia, Huntington's disease and Alzheimer's disease (Chang et al, 2012; Paoletti et al, 2013; Waxman and Lynch, 2005; Yamada, 2000). Thus, therapeutic approaches that modulate the activity and function of ionotropic glutamate receptors, so as to enhance normal synaptic functions while preventing pathological synaptic dysregulation, would aid in the treatment of multiple central nervous system disorders.

Medicinal plants are a source of many compounds that may exhibit capabilities to enhance cognition or prevent cognitive impairment. P. chinensis (Bunge) Regel (bai tou weng) is a common ingredient in several traditional Chinese medicines. The decoction of its rhizome is widely used to treat amoebic dysentery, vaginal trichomoniasis, and bacterial infection (Cheng et al, 2008; Ye et al, 1996; Zheng et al, 2010). This plant also exhibits sedative and analgesic effects, suggesting effects on the nervous system. A marker compound of $P$. chinensis, anemoside A3 (AA3), is a triterpenoid saponin implicated in relaxing renal arteries (Zhang et al, 2010) and protecting PC12 cells from apoptosis (Gao et al, 2003). In this study, we have investigated the beneficial effects of AA3 on synaptic and cognitive functions in normal adult mice.

\section{MATERIALS AND METHODS}

\section{Chemicals and Antibodies}

All chemicals were purchased from Sigma (St Louis, USA), unless stated otherwise. Antibodies specific for pAKT, AKT, $\operatorname{pCaMKII} \alpha, \mathrm{CaMKII} \alpha, \operatorname{pERK} 1 / 2$, and ERK $1 / 2$ were from Cell Signaling Technology (Beverly, USA); brain-derived neurotrophic factor (BDNF) antibody was from Santa Cruz (Dallas, USA); PSD-95 antibody was from Thermo Scientific Pierce; GluA1, pCREB, GluA2, and pSer831 GluA1 antibodies were from Millipore (Darmstadt, Germany);
pSer845 GluA1 antibody was from PhosphoSolutions (Aurora, USA); and actin antibody was from Sigma. The purity of AA3 was $>95 \%$ according to nuclear magnetic resonance analysis.

\section{Animal Behavioral Tests}

All animal experiments were approved by the Animal Care Committee of the Hong Kong University of Science and Technology. C57BL/6 (C57) mice (3-5 months old) were administered AA3 daily by oral gavage throughout the training in the Morris water maze (MWM) test. Six-week-old ICR mice were administered AA3 daily for 2 days before starting the forced swim test (FST). ICR mice (3-5 months old) were administered AA3 for 10 days before performing in the open field (OF) exploratory test and passive avoidance task (PAT). Eight-week-old Sprague-Dawley rats (300 g) were used for middle cerebral artery occlusion (MCAO); AA3 was administered $6 \mathrm{~h}$ after ischemia. Detailed procedures for animal behavioral tests are described in the Supplementary Information. Experimenters were blind to the experimental conditions in the animal behavioral tests.

\section{Statistical Analysis}

All data are expressed as arithmetic mean \pm SEM. Long-term potentiation (LTP) in hippocampal slices, miniature excitatory postsynaptic current (mEPSC) and dendritic spine density in cultured neurons, and BDNF level and protein expression of hippocampus were analyzed by unpaired Student's $t$-test. Neurotransmitter level in brain tissues, NMDA-evoked current and neuronal cell death in cultured neurons, cAMP level in hippocampal slices, neurological deficit evaluation of MCAO, and probe trial of MWM, PAT, FST, and motor activities in OF tests were analyzed by oneway ANOVA followed by Newman-Keuls post-hoc test. Paired pulse facilitation (PPF) in hippocampal slices, total distance moved in the $\mathrm{OF}$, and the acquisition training of MWM were analyzed by two-way repeated-measures ANOVA followed by post-hoc test. $P<0.05$ was considered statistically significant. All statistical analysis was performed using Graphpad prism v5.0.

Other experimental details are described in the Supplementary Information.

\footnotetext{
Figure I Anemoside A3 (AA3) facilitates synaptic transmission in mature adult mice. (a and b) AA3 did not affect the AMPA receptor (AMPAR)-mediated basal neurotransmission and paired pulse facilitation (PPF). (a) The input/output (I/O) curves were constructed from the relation between fiber volley (FV) amplitude and the fEPSP slope at the CA3-CAI synapses of hippocampal slices over a range of stimulus intensities. $n=1 \mid-12$ hippocampal slices from 4 brains. Control: fit with regression line $y=2.325 x+19.54, R^{2}=0.998$; AA3: fit with regression line $y=2.783 x+64.06, R^{2}=0.984$. (b) Graph showing PPF in control and AA3-treated mice. Paired presynaptic fiber stimulation pulses were delivered with varying interpulse intervals ranging from 20 to $400 \mathrm{~ms}$, to elicit postsynaptic responses. The plot summarizes the facilitation of the second fEPSP slope relative to the first one as a function of the inter-pulse interval. $n=4-5$ brains. (c) Enhancement of $\theta$-burst stimulation (TBS)-induced long-term potentiation (LTP) in hippocampal slices from 3-to 5-month-old AA3-treated mice. $n=1 \mid-12$ hippocampal slices from 4 brains. (d) Representative miniature excitatory postsynaptic current (mEPSC) traces of hippocampal neurons (I2 DIV) treated with DMSO (Control) or AA3 $(30 \mu \mathrm{M})$ for 2 days. Quantitative analysis of mEPSC frequency (e) and amplitude (f); $n=|9-2|$ neurons. ( $g$ ) Representative images of cultured hippocampal neurons treated with DMSO (Control) or AA3. Neuronal morphology was visualized by expressing GFP cDNA constructs in neurons. (h) Quantitative analysis of the numbers of total dendritic spines, mushroom-shaped spines, and filopodia in hippocampal neurons. $n=\mid 1-12$ neurons, $p<0.01$. (i-n) AA3 increases AMPAR subunit GluAI phosphorylation in the mouse hippocampus. Mice were orally administered AA3 daily for 2 days. AA3 increased GluAI phosphorylation at Ser83I (pS83I) or Ser845 (pS845) in the mouse hippocampus. Quantitative analysis of pS83 I GluAI/GluAI (i) and pS845 GluAI/GluAI (j). Quantitative analysis of total GluAI (k), GluA2 (I), PSD-95 (m), and actin ( $n$ ). $n=3-4$ brain tissues, *p<0.05 vs control (Cont).
} 
a

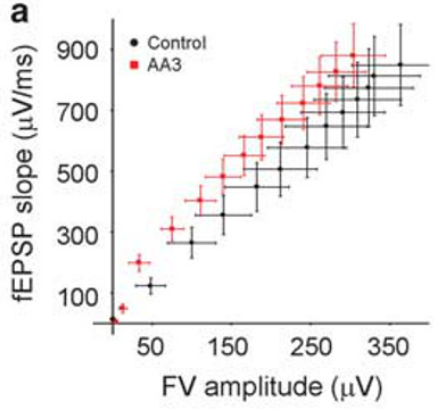

c

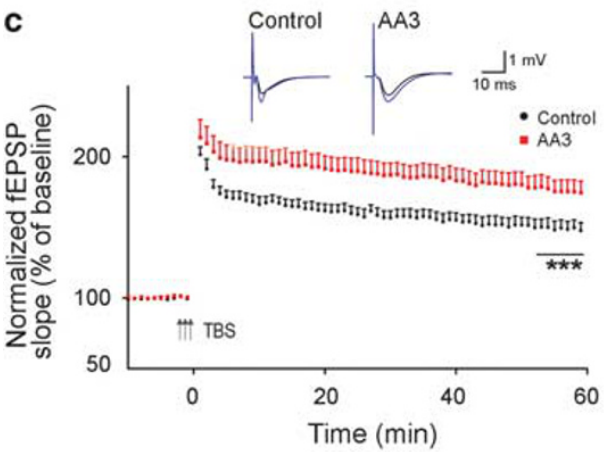

g
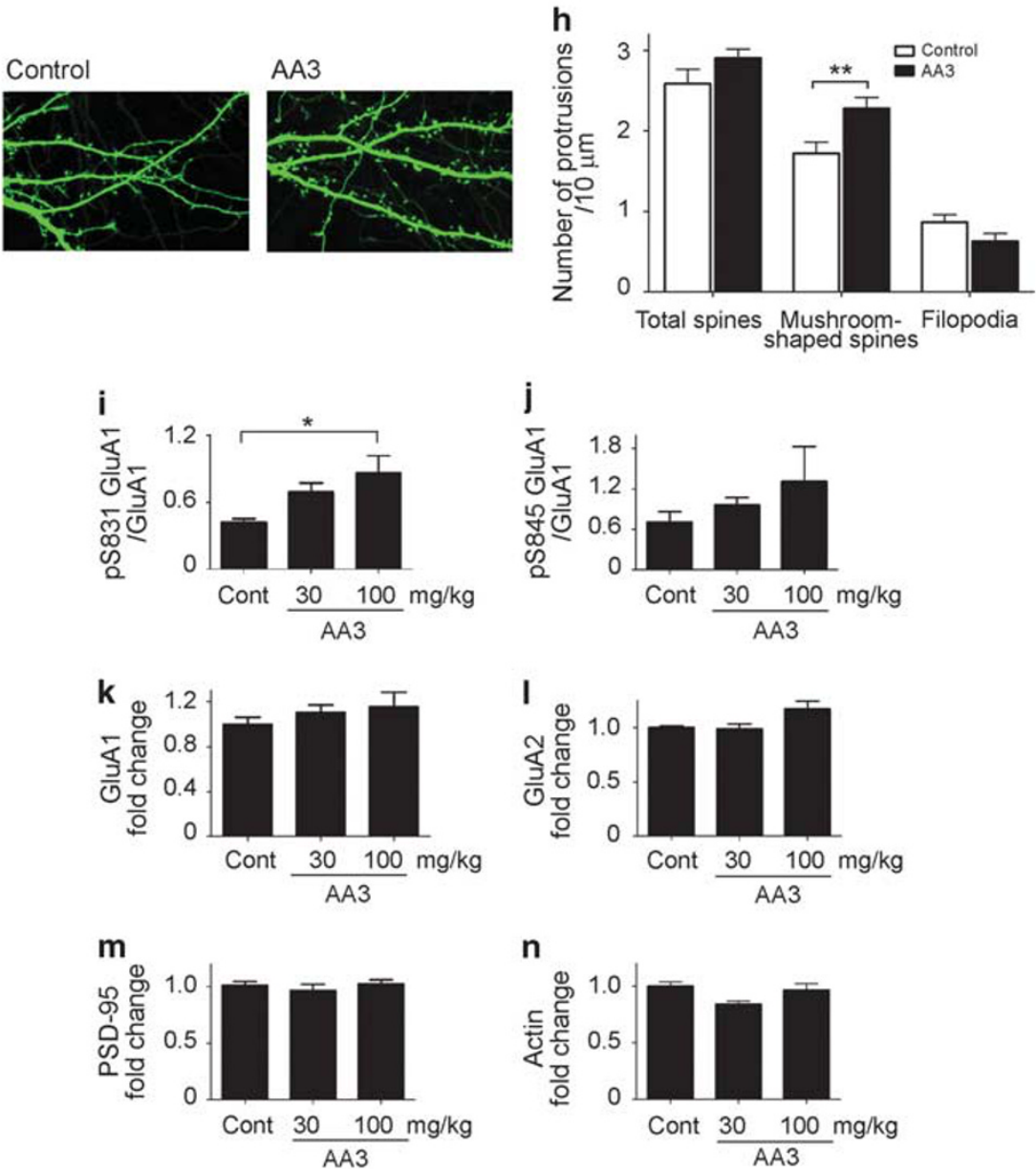


\section{RESULTS}

\section{AA3 Increases Functional Excitatory Synapses}

The suggested effects of $P$. chinensis on the nervous system (Gao et al, 2003) prompted us to investigate whether and how its major constituent, the AA3 compound, alters neuronal functions. As part of our effort to identify cognitive enhancers, we first determined the pharmacokinetics of AA3 in adult C57 mouse brain after a single-dose oral administration at $100 \mathrm{mg} / \mathrm{kg}$. Quantitative analysis of AA3 indicated that the compound was readily detected in both the plasma and the brain at 10 and $20 \mathrm{~min}$, respectively, after administration. The concentration of $\mathrm{AA} 3$ in the plasma peaked at $\sim 1 \mathrm{~h} \quad\left(C_{\max }=1673.1 \pm 209.0 \mathrm{ng} / \mathrm{ml}\right)$ and declined to $<300 \mathrm{ng} / \mathrm{ml}$ at $4 \mathrm{~h}$ after administration. In the brain, the level of AA3 peaked at $\sim 1.17 \mathrm{~h}\left(C_{\max }=129.5 \pm 47.8 \mathrm{ng} / \mathrm{g}\right)$ and decreased to $<30 \mathrm{ng} / \mathrm{g}$ at $3 \mathrm{~h}$ after administration (Supplementary Figure 1). After demonstrating that AA3 can enter the brain, we then examined whether the compound can potentiate synaptic functions in the adult mouse hippocampus, a brain region critical for spatial reference memory. Oral administration of AA3 $(100 \mathrm{mg} / \mathrm{kg})$ for 2 weeks did not affect the basal AMPA-mediated neurotransmission, as revealed by the fiber volley amplitude $v s$ fEPSP slope relation (Figure 1a), nor the short-term plasticity as revealed by the relatively unchanged PPF at Schaffer collateral fiber inputs onto CA1 pyramidal cells (Figure 1b; interaction: $\mathrm{F}_{(6,42)}=0.29, p=0.938$; drug effect: $\mathrm{F}_{(1,7)}=0.87$, $p=0.383)$. These findings suggest that AA 3 administration does not alter the basal synaptic strength and presynaptic function in mouse hippocampus. However, we showed that AA3 administration can potentiate the LTP formation in the hippocampal Schaffer collateral-CA1 pathway induced by $\theta$-burst stimulation (TBS), a cellular form of synaptic plasticity closely associated with learning and memory. TBS of the Schaffer collateral inputs to CA1 pyramidal neurons induced stable LTP in mouse hippocampal slices as shown by the persistent increase in fEPSP slope for at least $60 \mathrm{~min}$ (Figure 1c). Interestingly, the mice that received oral administration of AA3 and trained in the MWM for 2 weeks demonstrated an enhanced LTP (by $18.2 \pm 0.5 \%, p<0.001$; Figure 1c) when compared with the mice with MWM training alone. These findings collectively suggest that AA3 enhances synaptic plasticity and may benefit memory formation in the adult mouse.

Next, we examined whether AA3 potentiates synaptic functions by regulating the structural or functional connectivity of hippocampal neurons. AA3 treatment significantly increased the frequency of AMPAR-mediated mEPSC $(\sim 47 \%$ increase, $p<0.05$; Figure $1 \mathrm{~d}-\mathrm{f})$ in dissociated cultured hippocampal neurons, indicating enhanced basal excitatory synaptic transmission. Moreover, a slight but significant increase in mEPSC amplitude was observed in AA3-treated neurons (by $\sim 14 \%, p<0.05$; Figure 1f). These results suggest that AA3 can increase the number of functional excitatory synapses and AMPAR abundance within individual synapses in cultured hippocampal neurons. Concordant with these functional enhancements, AA3-treated hippocampal neurons also exhibited more mushroom-shaped dendritic spines (Figure $1 \mathrm{~g}$ and $\mathrm{h}$ ), suggesting that AA3 can promote the structural changes of synapses.

\section{AA3 Increases the Phosphorylation of AMPAR Subunit GluA1 in the Mouse Hippocampus}

AA3's facilitation of synaptic function was further characterized by examining the expressions of synaptic proteins and downstream signaling molecules in adult mice that had received oral administration of AA3. The abundance of AMPARs at synapses in hippocampal neurons is correlated with synaptic strength and thus hippocampal-dependent learning (Anggono and Huganir, 2012; Riedel et al, 2003). Synaptic expression of AMPARs can be controlled by their phosphorylationdependent insertion/removal from synapses (Lee et al, 2000; Shepherd and Huganir, 2007). For example, the phosphorylation of AMPAR subunit GluA1 at Ser845 by PKA (Esteban et al, 2003) and at Ser831 by CaMKII (Mammen et al, 1997) are important for GluA1-containing AMPAR insertion into the extrasynaptic surface and translocation to synapses, respectively (Derkach et al, 2007; Lee et al, 2003). Therefore, we examined whether AA3 regulates the synaptic localization of AMPARs through GluA1 phosphorylation. Indeed, AA3 administration increased GluA1 phosphorylation at Ser831 and Ser845 in the mouse hippocampus $(\sim 100$ and $80 \%$ increases, respectively, at $100 \mathrm{mg} / \mathrm{kg}$; Figures $1 \mathrm{i}$ and j, and Supplementary Figure 2), whereas the total protein levels of the receptors remained relatively unchanged (Figure $1 \mathrm{k}$ and 1 , and Supplementary Figure 2). These findings suggest that AA3 potentiates neurotransmission and synaptic plasticity in the hippocampus via an AMPARdependent mechanism. The increase of GluA1 phosphorylation and its function in mice by AA3 may be specific to the hippocampus. AA3 treatment resulted in an increase in both the phosphorylated GluA1 protein and total GluA protein in the prefrontal cortex (Supplementary Figure 3). Normalizing the level of phosphorylated GluA1 protein with total protein showed no enhancement of GluA1 phosphorylation between different drug treatment conditions (Supplementary Figure 3). These findings collectively suggest that AA3 enhances synaptic transmission and plasticity in the hippocampus, possibly through the modulation of AMPAR functions.

\section{AA3 Increases Monoamine Neurotransmitter Levels}

Monoamine neurotransmitters such as 5-HT and NE have significant roles in modulating various physiological functions including mood and cognitive functions (Cahill et al, 1994; Geyer and Vollenweider, 2008; Normann and Clark, 2005). In particular, NE was recently shown to facilitate the induction of CA3-CA1 LTP and hippocampus-dependent contextual memory by increasing GluA1 phosphorylation (Hu et al, 2007). Therefore, we sought to determine whether AA3 regulates the expression of neuromodulators that influence synaptic plasticity in the hippocampus. AA3 administration increased 5-HT and NE levels in both the mouse hippocampus and prefrontal cortex (Table 1). Nonetheless, AA3 administration ( 30 and $100 \mathrm{mg} / \mathrm{kg}$ ) did not change monoamine oxidase activity in the mouse hippocampus (MAO-A: $\mathrm{F}_{(2,9)}=0.100, p=0.905$; and MAO-B: $\mathrm{F}_{(2,9)}=0.743, p=0.503 ; n=4$ brains), suggesting that the effect of AA3 on monoamine neurotransmitter levels is not due to the blockade of their degradation. Therefore, AA3 may also influence synaptic connectivity and strength by increasing the presence of monoamine neurotransmitters. 
Table I AA3 Administration Increases Amounts of 5-HT, 5-HIAA, and NE in the Mouse Hippocampus (a) and Prefrontal Cortex (b)

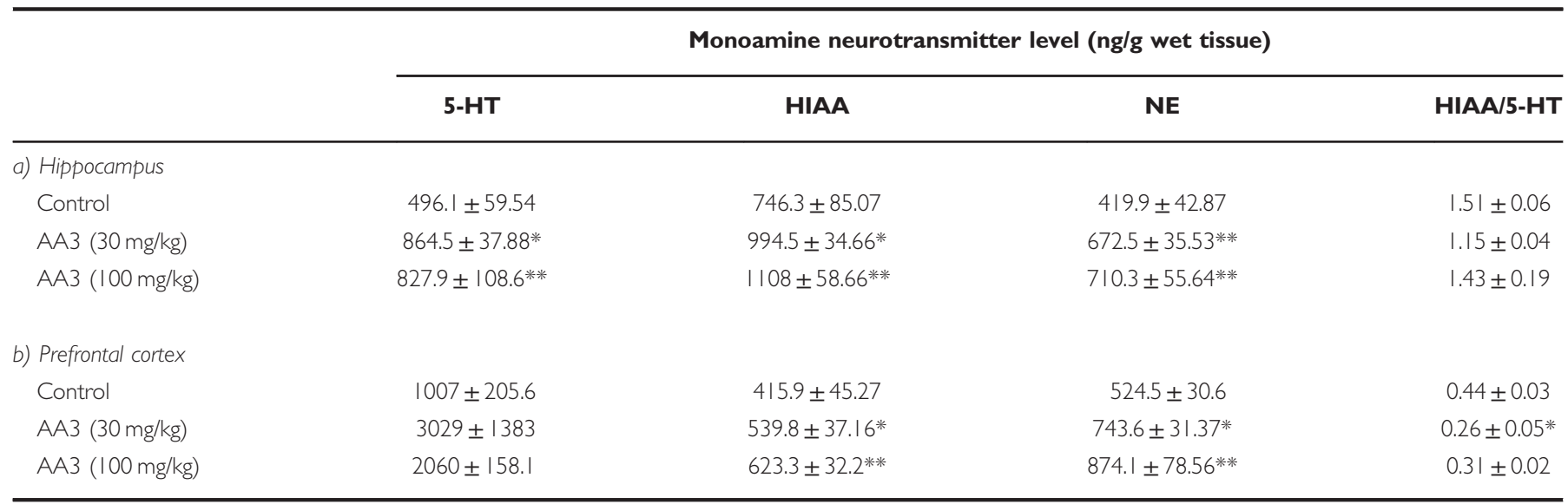

Abbreviation: AA3, Anemoside A3.

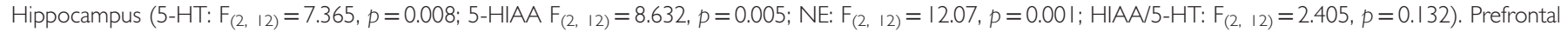

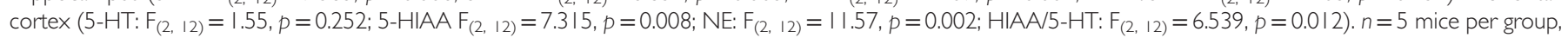
$* p<0.05$, ** $p<0.01$ vs Control.

\section{AA3 Enhances Activation of Synaptic Signaling Pathway}

Long-term memory formation requires the induction of gene expression, which is coordinately regulated by various synaptic signaling molecules such as cAMP, CaMKII, AKT, and ERK1/2; these facilitate CREB-dependent gene transcription and the subsequent modulation of synaptic plasticity (Waltereit and Weller, 2003). To determine whether AA3 facilitates LTP by modifying the phosphorylation and activation states of such molecules, the effects of its administration on the activation of various associated proteins were investigated. Indeed, direct in vitro treatment of AA3 increased ERK1/2 phosphorylation (Figure 2a and $\mathrm{b}$, and Supplementary Figure 4a) and cAMP production (Figure $2 \mathrm{c} ; \mathrm{F}_{(2,6)}=5.63 ; p=0.042$ ) in acute rat hippocampal slices. Furthermore, in vivo oral administration enhanced the phosphorylation of CREB (Figure 2d, and Supplementary Figure $4 \mathrm{~b}$ ) and various kinases including ERK1/2, CaMKII $\alpha$, and AKT (Figure 2e-h, and Supplementary Figure 4c) in the adult mouse hippocampus. Again, the regulation of these signal activations by AA3 was not observed in the prefrontal cortex of AA3-treated animals, suggesting that AA3 may have specific roles in the mouse hippocampus (data not shown). BDNF, a neurotrophin family member, is a CREB target gene. BDNF is crucial for hippocampal synaptic plasticity regulation and long-term memory formation (Minichiello, 2009). BDNF protein levels increased significantly in the adult mouse hippocampus following AA3 administration (100 mg/kg) for 2 days (by $9.5 \pm 0.03 \%, n=8, p<0.01$ ), as determined by ELISA. Furthermore, western blot analysis confirmed an increase in mature BDNF levels in the adult mouse hippocampus following AA3 administration (Figure 2i and Supplementary Figure 4d). These findings highlight the variety of molecules and signaling pathways integral to memory and cognition that are modulated by AA3.

\section{AA3 Is an NMDAR Modulator}

Although the various mechanisms by which AA3 influences AMPAR signaling are crucial to the cognition-enhancing properties of the compound, interaction with the NMDAR might indicate a neuroprotective property as well. The indication of $P$. chinensis on analgesic activity prompted us to speculate that AA3 also modulates NMDARs. Thus, we measured the whole-cell recordings from cultured hippocampal neurons, which showed that AA3 inhibited the NMDA-evoked current at a holding potential of $-50 \mathrm{mV}$. This inhibition increased with increasing NMDA concentration (Figure $3 \mathrm{a}$ and $\mathrm{b} ; \mathrm{F}_{(2,30)}=9.635 ; p=0.0006$ ), required the presence of NMDA (Figure 3c), and was mediated through the inhibition of NMDA-induced $\mathrm{Ca}^{2+}$ influx (Figure 3d). Moreover, AA3 similarly inhibited the NMDAevoked current at both negative and positive membrane potentials ( $\mathrm{IC}_{50}$ : 18.48 and $20.19 \mu \mathrm{M}$ at -50 and $+50 \mathrm{mV}$, respectively; Figures $3 e$ and $f$ ), suggesting that NMDAR inhibition by AA3 is independent of membrane potential (ie, voltage).

Given our observation on the effect of AA3 as an NMDAR modulator, we examined whether AA3 exerts neuroprotective effects against glutamate excitotoxicity and ischemic insult. AA3 treatment significantly reduced NMDA stimulationinduced death of cultured hippocampal neurons (Figure 3g; $\left.\mathrm{F}_{(3,12)}=8.081 ; p=0.003\right)$, suggesting that AA3 protects neurons from excitotoxicity by blocking excessive NMDAR stimulation. Furthermore, AA3 administration significantly reduced the extent of ischemic injury as evidenced by the decreased infarct volume $\left(\mathrm{F}_{(3,38)}=4.922, p=0.006\right)$ and neurological deficit scores $\left(\mathrm{F}_{(3,38)}=3.579, p=0.023\right)$ in the MCAO ischemic model (Figure $3 \mathrm{~h}-\mathrm{k}$ ). These results collectively demonstrate that AA3 is a non-competitive NMDAR modulator that protects neurons from excitotoxicity.

\section{AA3 Improves Cognitive Functions}

The mechanistic effects of AA3 on both AMPARs and NMDARs suggest AA3 is a potential cognitive enhancer. Thus, it has the potential to improve learning and memory, while also protecting neurons. We therefore investigated whether AA3 improves cognitive function in naive adult 
mice. To this end, we assessed the cognitive performance of mice aged 3-5 months, an age at which they have completed development but not yet begun senescence. We compared learning and memory between AA3- and vehicle-administered mice in the OF (Figure 4a), MWM (Figure 4b), and PAT (Figure 4a) tests, as well as examined changes in depression-like behavior using the FST. In the first day of OF training, AA3 administration did not significantly alter locomotor activity (Supplementary Figure 5a; $\mathrm{F}_{(2,57)}=$ $0.041, p=0.96)$ or change the preference for the peripheral zone of the arena (Supplementary Figure $5 b$ and c), suggesting that AA3 does not affect locomotor activity. The vehicle- and AA3-treated mice exhibited normal habituation to the novel environment, as indicated by the decrease in
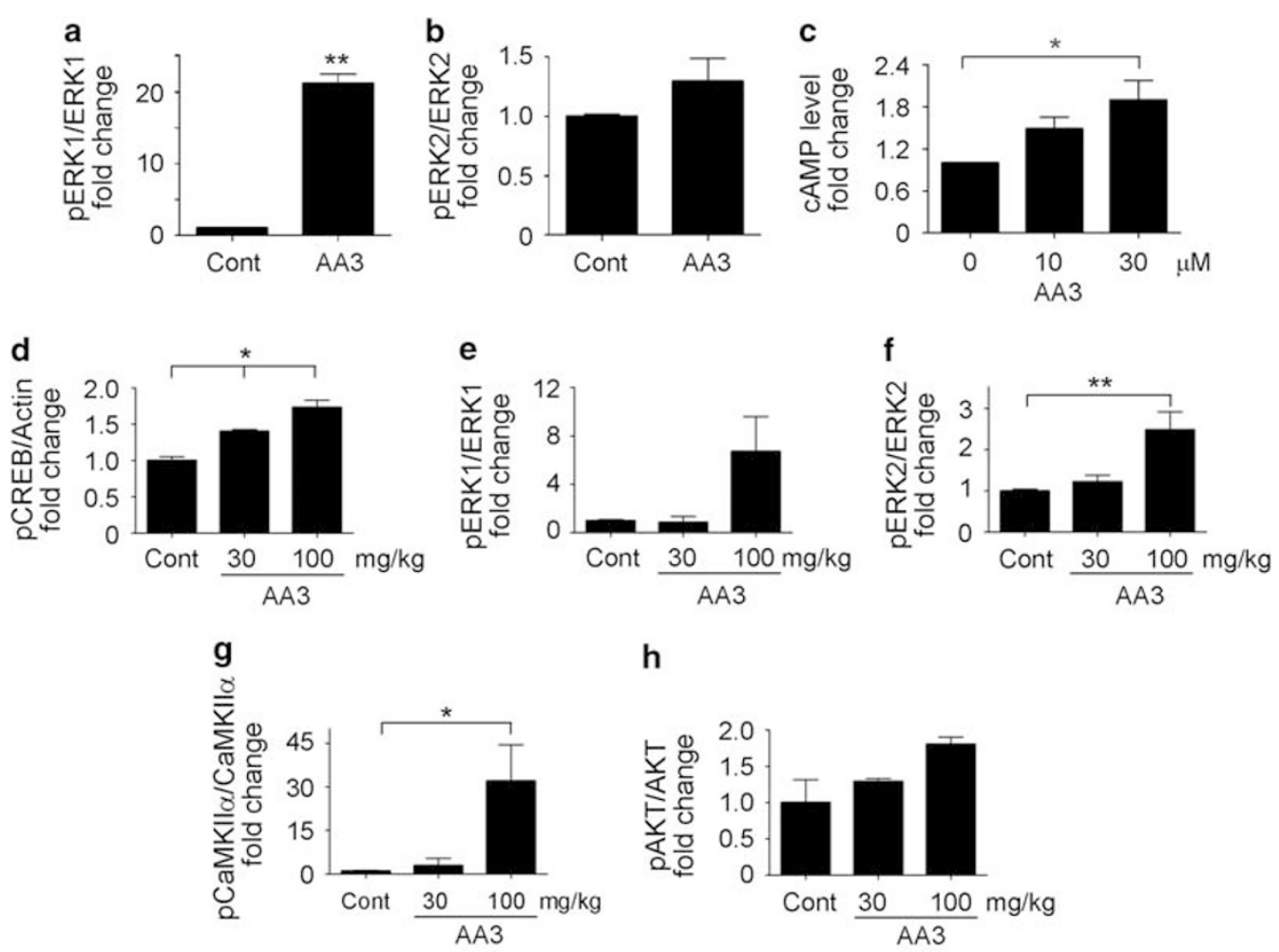

h
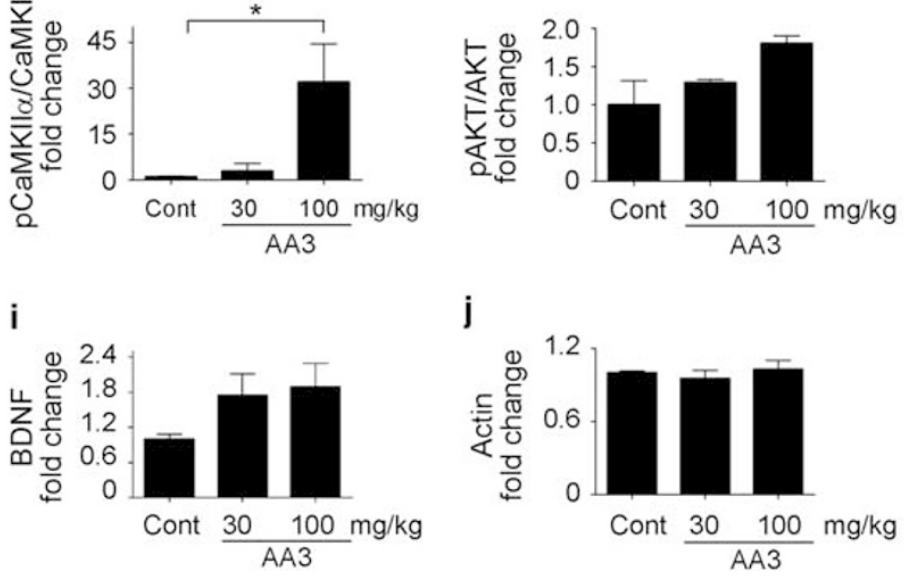

Figure 2 Anemoside $A 3$ (AA3) enhances signaling proteins activation in the mouse hippocampus. Treatment with $A A 3(30 \mu M)$ increased ERKI (a) and ERK2 (b) phosphorylation in acute rat hippocampal slices. (c) AA3 increased cAMP levels in acute hippocampal slices. $n=3$; $* 2<0.05$ vs $0 \mu M$. (d-h) AA3 ( 100 mg/kg) enhanced CREB, ERKI/2, CaMKIl $\alpha$, and AKT phosphorylation in the mouse hippocampus. Mice were orally administered AA3 daily for 2 days. Ratios of pCREB/actin (d), pERKI/ERKI (e), pERK2/ERK2 (f), pCaMKIl $\alpha / C a M K I l \alpha(g)$, and pAKT/AKT (h). (i and j) AA3 increased brain-derived neurotrophic factor (BDNF) but not actin protein levels in the mouse hippocampus. $n=4$ mouse brains, $* p<0.05, * * p<0.005$ vs control (Cont).

Figure 3 Anemoside $A 3$ (AA3) protects against NMDA receptor (NMDAR)-mediated excitotoxicity through the direct modulation of NMDAR. (a-f) AA3 is a non-competitive NMDAR modulator. (a) Current traces and (b) quantitative analysis of AA3-dependent inhibition of NMDA-evoked current in hippocampal neurons at a holding potential of $-50 \mathrm{mV}$. Inhibition of NMDA-evoked current by AA3 increased with increasing concentrations of NMDA. $n=4-5, * p<0.05$, **** $p<0.005$ vs $10 \mu \mathrm{M}$. (c) The presence of NMDA $(200 \mu \mathrm{M})$ was required for the blockade effect of AA3 (30 $\mu$ M) on NMDAR. Current traces are shown. (d) AA3 inhibited the NMDA-induced calcium influx in cultured hippocampal neurons. (e) Current traces showing the blockade effect of AA3 on NMDA-evoked current at holding potentials of -50 and $+50 \mathrm{mV}$. (f) Concentration-response curves showing the inhibition of NMDA-evoked current by AA3 at holding potentials of -50 and $+50 \mathrm{mV}$. $n=5-1 \mathrm{l}$. (g) AA3 protected cultured hippocampal neurons from NMDA-induced excitotoxicity $(200 \mu M) . n=4$ experiments, $* * *<0.01$ vs $0 \mu M$. ( $h-k)$ AA3 protected against ischemic brain injury in adult rats. (h) Schematic diagram illustrates the time line of AA3 administration and tissue collection in middle cerebral artery occlusion (MCAO). AA3 was orally administered to rats at $6 \mathrm{~h}$ after MCAO. (i) Representative images of brain slices stained with TTC. TTC-stained red regions indicate unaffected tissue and pale white regions show infarcted tissue. AA3 reduced infarct volume $(j)$ and neurological deficit scores $(k)$ in rats under ischemic injury. $n=8-10, * p<0.05$, *** $p<0.0$ I vs control (Cont). 

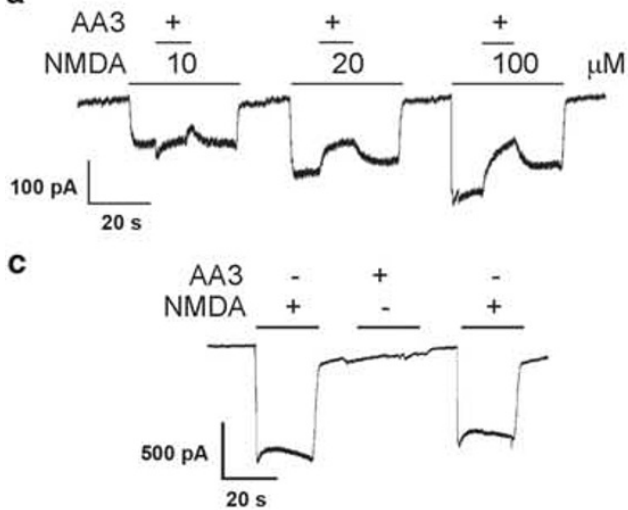

e

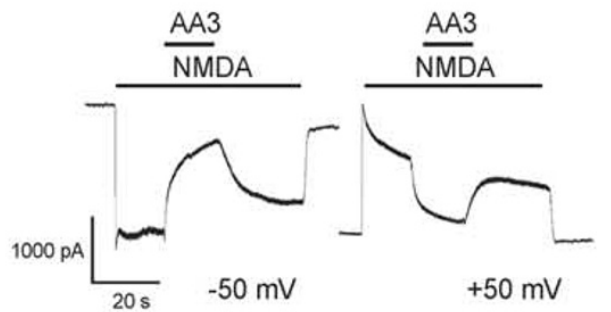

f

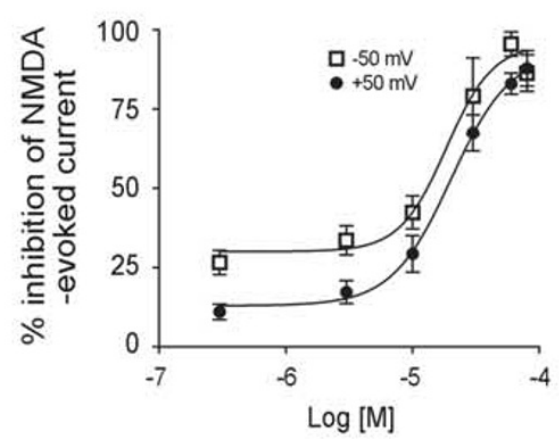

h
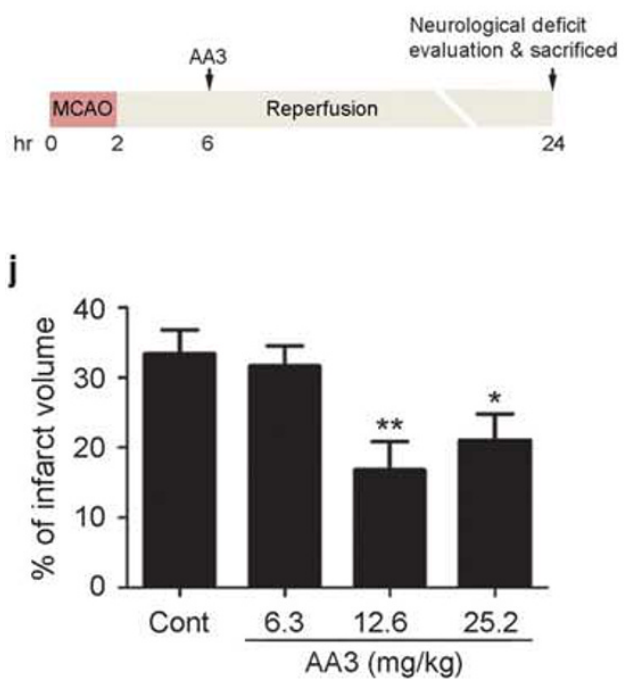

b

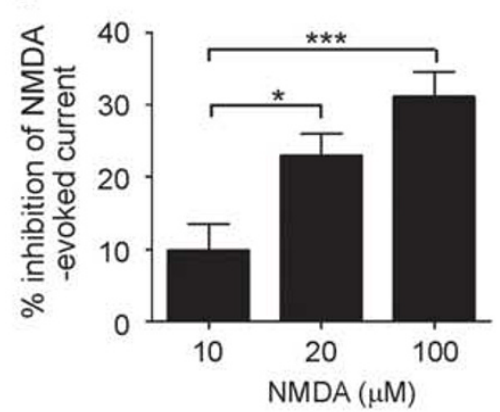

d

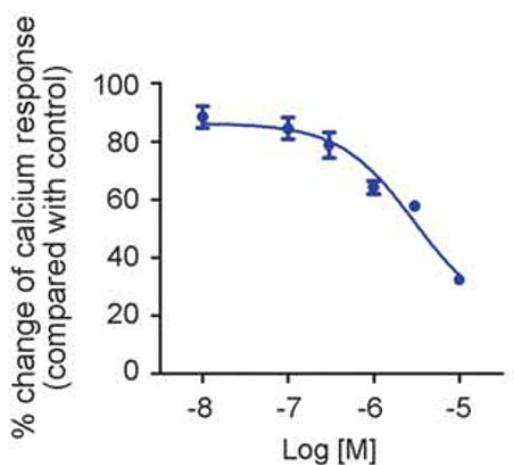

g

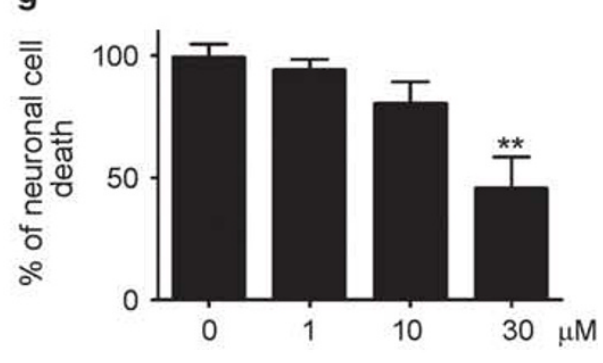

i
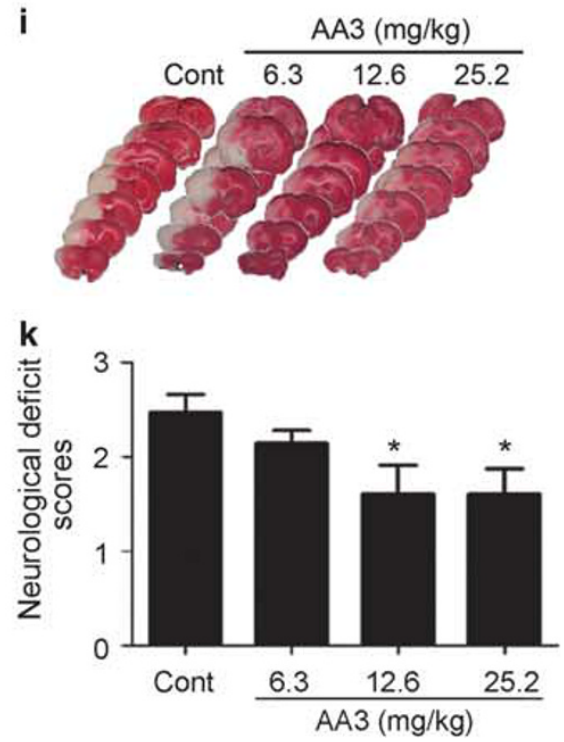
a
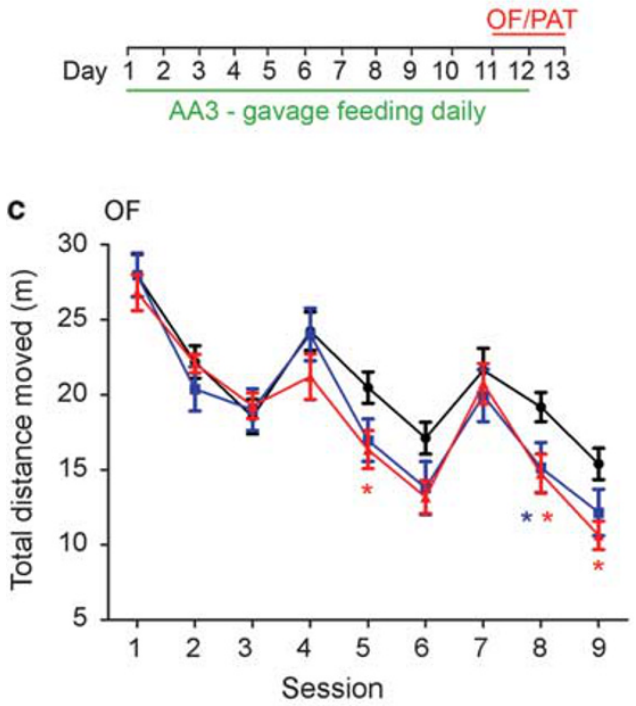

e

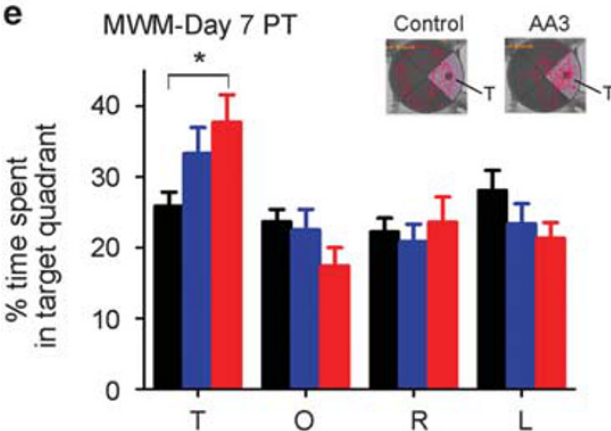

g PAT

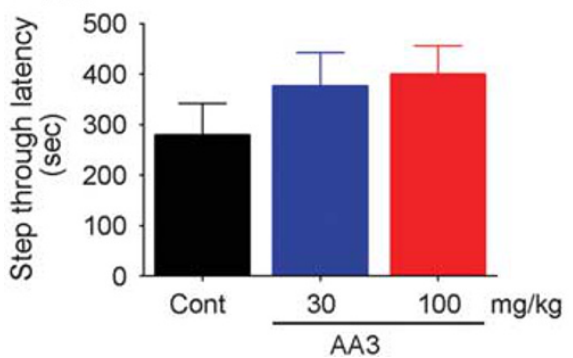

b

Water maze training daily 2 trials/day

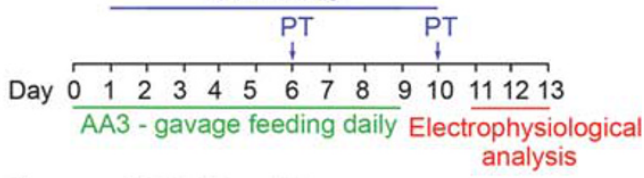

d

MWM-Acquisition

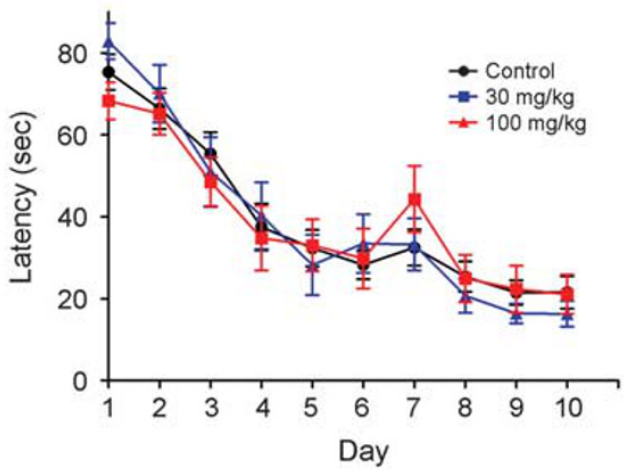

f MWM-Day $10 \mathrm{PT}$

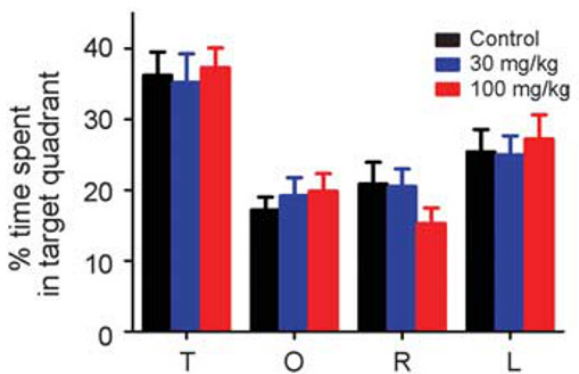

$\mathbf{h}_{\text {FST }}$

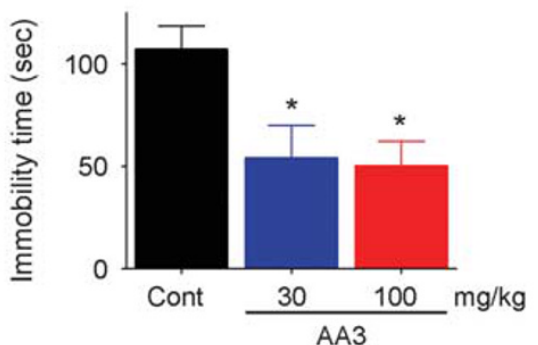

Figure 4 Anemoside $A 3$ ( $A A 3$ ) improves learning and memory performance in mice. (a and b) Schematic diagrams illustrate the timeline of $A A 3$ administration and behavioral experiments including OF/PAT (a) and MWM (b). (c) Effect of AA3 in the OF. Mice were administered AA3 for I0 days and then allowed to explore a novel arena for 3 consecutive days; each day, training lasted $15 \mathrm{~min}$. The graph is expressed as the distance travelled within each 5-min session. $n=18-24$ mice per group. *Red, the difference between control and $100 \mathrm{mg} / \mathrm{kg}$; *blue, the difference between control and $30 \mathrm{mg} / \mathrm{kg}$ at $p<0.05$ at indicated sessions. ( $d-f)$ AA3 improved spatial reference memory in the MWM test. (d) Escape latencies of mice during the course of training. (e) AA3-treated $(100 \mathrm{mg} / \mathrm{kg}$ ) mice learned the location of the submerged platform faster than the control mice. AA3-administered mice (I $00 \mathrm{mg} / \mathrm{kg})$ acquired spatial memory on day 7 as reflected by the increased time they spent in the $T$ zone $\left(F_{(2.44)}=4.629, p=0.015 ; * p<0.05,100 \mathrm{mg} / \mathrm{kg}\right.$ vs Control). T, target quadrant; L, O, R, the left, opposite, and right of the target quadrant, respectively. $n=1 \mid$ - 22 mice per group. Typical swimming traces on the day 7 probe trial showing the effect of AA3 (100 mg/kg) on spatial memory (upper-right inset). ( $\mathrm{f}$ ) The probe trial result revealed that the control mice acquired spatial memory on day 10. (g) AA3 did not affect the performance in the PAT. Mice were administered AA3 for 10 days before testing. $n=10$ mice per group. (f) AA3 exhibited an antidepressant-like effect. Mice, orally administered with AA3 for 2 days, were subjected to the FST. AA3 reduced the immobility time of mice. $n=10$ per group. ${ }^{*} p<0.05$ vs control (Cont).

total moving distance across trainings (Figure 4c and Supplementary Figure 5a). Compared with the control, mice administered $100 \mathrm{mg} / \mathrm{kg}$ AA3 exhibited a quicker habituation to the novel environment, as revealed by reduced locomotion over the course of training (Figure $4 \mathrm{c}(100 \mathrm{mg} / \mathrm{kg}$ vs Control: interaction, $\mathrm{F}_{(8,320)}=2.86, p=0.004$; drug effect: $\mathrm{F}_{(1,40)}=3.77$, $p=0.059$; session: $\left(\mathrm{F}_{(8,320)}=50.42, p<0.0001\right) ;(30 \mathrm{mg} / \mathrm{kg} v \mathrm{~s}$ Control: interaction, $\mathrm{F}_{(8,320)}=1.85, p=0.0673$; drug effect: $\mathrm{F}_{(1,40)}=1.57, p=0.2171$; session: $\left.\mathrm{F}_{(8,320)}=49.05, p<0.0001\right)$ and Supplementary Figure 5a). Likewise, the MWM test 
demonstrated that AA3 improved memory formation: AA3 did not affect the acquisition of learning of a hidden platform location over the course of the 10-day training (Figure $4 \mathrm{~d}$; interaction: $\mathrm{F}_{(18,396)}=0.599, p=0.901$; drug effect: $\mathrm{F}_{(2,44)}=$ $0.008, p=0.992)$. However, AA3-treated mice acquired spatial memory more rapidly (Figures $4 \mathrm{e}$ and $\mathrm{f}$ ) and were able to identify the correct quadrant from which the hidden platform was removed in the day-7 probe trial (Figure 4e; Control: $F_{(3,84)}=13.99, p=0.249 ; 30 \mathrm{mg} / \mathrm{kg}: \mathrm{F}_{(3,40)}=3.526$, $\left.p=0.023 ; 100 \mathrm{mg} / \mathrm{kg}: \mathrm{F}_{(3,52)}=13.99, p=0.0002\right)$. In contrast, the control mice were unable to locate the correct quadrant until day 10, the time when all the groups were able to learn the location of the platform (Figure 4f; Control: $\mathrm{F}_{(3,84)}=$ 8.106, $\quad p<0.0001 ; \quad 30 \mathrm{mg} / \mathrm{kg}: \quad \mathrm{F}_{(3,40)}=5.765, \quad p=0.002$; $\left.100 \mathrm{mg} / \mathrm{kg}: \mathrm{F}_{(3,52)}=12.05, p<0.0001\right)$. The AA3-treated mice did not enhance memory formation in the PAT (Figure $4 \mathrm{~g}$; $\left.\mathrm{F}_{(2,27)}=1.057 ; p=0.3613\right)$. These behavioral results collectively suggest that AA3 may have beneficial effect on spatial reference memory. In addition to the evidence supporting AA3's memory-enhancement function, AA3-treated mice also exhibited significantly reduced immobility in the FST (Figure $4 \mathrm{~h} ; \mathrm{F}_{(2,27)}=5.652 ; p=0.009$ ), suggesting that AA3 may have an antidepressant-like effect.

\section{DISCUSSION}

Memory formation involves the rearrangement of synaptic connections within the neural circuitry. Many factors including neuronal excitability, synaptic plasticity, and neuronal survival modulate neural circuitry functioning. The precise control of the activity and functions of ionotropic glutamate receptors is critical for proper synaptic transmission and synaptic plasticity (Riedel et al, 2003). Here we report that AA3, a triterpenoid saponin isolated from $P$. chinensis, has multiple effects that modulate the functions of glutamatergic systems. On the one hand, AA3 can potentiate AMPAR functions by modulating receptor trafficking during learning and memory processes; on the other hand, it prevents NMDAR overactivation by directly blocking the channel during neuronal excitotoxicity. AA3 regulates multiple neural plasticity-related signaling pathways and likely exerts its memory-enhancement effect by increasing LTP in the hippocampus.

AMPAR function is regulated by the abundance, combination, and properties of AMPAR subunits at synaptic sites. Identifying positive allosteric modulators of AMPARs is a potential strategy for developing therapeutics against agingor disease-related cognitive impairments (Yamada, 2000). For example, ampakines are a family of drugs that bind directly to AMPARs and enhance their functions by slowing the receptor deactivation and desensitization, resulting in a prolonged synaptic response (Lynch, 2006). The present study shows that AA3 facilitates AMPAR function by modulating the phosphorylation status of AMPAR subunits. GluA1 serine phosphorylation regulates AMPAR trafficking, which is important for modulating synaptic strength and thus memory formation. GluA1 serine phosphorylation can be enhanced by multiple kinases including CaMKII $\alpha$, PKA, and ERK1/2 on NMDAR activation; these synaptic signaling pathways can in turn be modulated by monoamine neurotransmitters such as NE and 5-HT (Esteban et al, 2003;
Hu et al, 2007; Jitsuki et al, 2011; Mammen et al, 1997). Given that NE and 5-HT levels in the hippocampus were elevated after AA3 administration, it is tempting to speculate that increased monoamine neurotransmitter presence is one of the mechanisms underlying the AA3-stimulated increased phosphorylation and synaptic expression of AMPARs.

Long-term memory formation requires gene transcription. CREB is the key transcription factor essential for long-term changes in synaptic strength and plasticity (Greer and Greenberg, 2008). Its activation is important for strengthening synaptic connections by enhancing the transcription of synaptic proteins in response to environmental changes. CREB activation is induced after increased $\mathrm{Ca}^{2+}$ influx on NMDAR activation and is modulated by various signaling cascades. These cascades include elevated intracellular cAMP triggered by the activation of G-protein-coupled receptors (GPCRs), receptor tyrosine kinases, and/or monoamine neurotransmitter receptors (Greer and Greenberg, 2008). Thus, increasing CREB activation is another potential therapeutic strategy for developing cognitive enhancers (Tully et al, 2003). Whether AA3 triggers the activation of cAMPdependent or other synaptic signaling pathways via GPCR stimulation awaits further investigation. Nonetheless, the AA3-induced cellular and molecular modifications in the brain are associated with improved memory performance.

In addition to being crucial for overall neural health, neuronal survival can be specifically beneficial for modulating neuronal connectivity and synaptic transmission strength. Therefore, homeostatic maintenance of NMDAR activity is critical for the maintenance of neuronal health and cognitive functions. Under physiological conditions, NMDAR is important in mediating synaptic plasticity via calciummediated signaling pathways (Rebola et al, 2010). However, NMDAR hyperactivation under pathological conditions leads to excessive $\mathrm{Ca}^{2+}$ influx into neurons, resulting in excitotoxicity (Talantova et al, 2013; Waxman and Lynch, 2005). Thus, it would be desirable to develop an 'optimal' NMDAR modulator that blocks 'excessive' receptor activity, especially during prolonged depolarization, but exerts little effect under normal physiological conditions. Most identified NMDAR antagonists such as MK801 do not fall into this category, because they perturb normal neurotransmission and cause cognitive impairment in normal subjects (Lukoyanov and Paula-Barbosa, 2000; Manahan-Vaughan et al, 2008). Another NMDAR antagonist, memantine, which is used as a therapeutic drug for Alzheimer's disease, enhances cognitive function. As a low-affinity NMDAR antagonist, memantine selectively blocks the hyperactivated NMDAR under pathological conditions (Muller et al, 1995; Parsons et al, 2007), a feature that may underlie its beneficial effect on cognitive function. Here we show that AA3 is also a lowaffinity non-competitive NMDAR modulator that inhibits NMDAR activity with slow on-rate inhibition and voltageindependent properties (Figure 3). These properties allow the compound to protect neurons against neurological insults under prolonged depolarization with minimal impact on normal neuronal activity, suggesting that AA3 is a potential therapeutic candidate for protecting against neurodegeneration and alleviating cognitive dysfunctions. Finally, the NMDAR antagonist ketamine recently demonstrated antidepressant-like effects. Its administration increased synapse formation and exhibited an antidepressant-like effect 
(Li et al, 2010). Consistent with the antagonistic effect of AA3 on NMDAR function, AA3-treated mice exhibited improved performance in the FST, raising another possibility that AA3 can be developed as a potential therapeutic target for depression.

In conclusion, AA3 is a natural triterpenoid saponin that enhances synaptic plasticity in the adult mouse hippocampus and facilitates spatial memory in adult mice. Thus, this small molecule may have beneficial effects on cognitive impairment and neurodegenerative disorders such as Alzheimer's disease. AA3 is a compelling subject for further study as well as further development for various clinical applications.

\section{FUNDING AND DISCLOSURE}

The authors declare no conflict of interest.

\section{ACKNOWLEDGMENTS}

We are grateful to Dr Guangmiao Fu, Dr Yuming Zhao, Dr Yu Pong Ng, Dr Yu Chen, Venus WS Lee, Ying Dai, Ke Gong, Cara Kwong, and Busma Butt for their excellent technical assistance. We thank Dr Amy KY Fu and Sejal M Mody for the critical reading of the manuscript. We are grateful to Professor Nikolaus Sucher, Professor Wing-Ho Yung, Dr Florian Plattner, and members of the Ip laboratory for many helpful discussions. This study was supported in part by the National Key Basic Research Program of China (2013CB530900), the Hong Kong Research Grants Council Theme-based Research Scheme (T13-607/12R), the Research Grants Council of Hong Kong SAR (HKUST 660810, 661013, and 661111), the Innovation and Technology Commission of Hong Kong SAR (ITS/179/11FP), the Hong Kong Jockey Club Charities Trust, the Shenzhen Peacock Plan, and the SH Ho Foundation.

\section{REFERENCES}

Anggono V, Huganir RL (2012). Regulation of AMPA receptor trafficking and synaptic plasticity. Curr Opin Neurobiol 22: 461-469.

Cahill L, Prins B, Weber M, McGaugh JL (1994). Beta-adrenergic activation and memory for emotional events. Nature 371: 702-704.

Chang PK, Verbich D, McKinney RA (2012). AMPA receptors as drug targets in neurological disease-advantages, caveats, and future outlook. Eur J Neurosci 35: 1908-1916.

Cheng L, Zhang M, Zhang P, Song Z, Ma Z, Qu H (2008). Silver complexation and tandem mass spectrometry for differentiation of triterpenoid saponins from the roots of Pulsatilla chinensis (Bunge) Regel. Rapid Commun Mass Spectrom 22: 3783-3790.

Derkach VA, Oh MC, Guire ES, Soderling TR (2007). Regulatory mechanisms of AMPA receptors in synaptic plasticity. Nat Rev Neurosci 8: 101-113.

Esteban JA, Shi SH, Wilson C, Nuriya M, Huganir RL, Malinow R (2003). PKA phosphorylation of AMPA receptor subunits controls synaptic trafficking underlying plasticity. Nat Neurosci 6: 136-143.

Gao XD, Ye WC, Yu AC, Zhang Y, Tan RX, Li M et al (2003). Pulsatilloside A and anemoside A3 protect PC12 cells from apoptosis induced by sodium cyanide and glucose deprivation. Planta Med 69: 171-174.
Geyer MA, Vollenweider FX (2008). Serotonin research: contributions to understanding psychoses. Trends Pharmacol Sci 29: 445-453.

Greer PL, Greenberg ME (2008). From synapse to nucleus: calciumdependent gene transcription in the control of synapse development and function. Neuron 59: 846-860.

$\mathrm{Hu}$ H, Real E, Takamiya K, Kang MG, Ledoux J, Huganir RL et al (2007). Emotion enhances learning via norepinephrine regulation of AMPA-receptor trafficking. Cell 131: 160-173.

Jitsuki S, Takemoto K, Kawasaki T, Tada H, Takahashi A, Becamel C et al (2011). Serotonin mediates cross-modal reorganization of cortical circuits. Neuron 69: 780-792.

Kawahara Y, Ito K, Sun H, Aizawa H, Kanazawa I, Kwak S (2004). Glutamate receptors: RNA editing and death of motor neurons. Nature 427: 801.

Kumar J, Mayer ML (2013). Functional insights from glutamate receptor ion channel structures. Annu Rev Physiol 75: 313-337.

Lee HK, Barbarosie M, Kameyama K, Bear MF, Huganir RL (2000). Regulation of distinct AMPA receptor phosphorylation sites during bidirectional synaptic plasticity. Nature 405: 955-959.

Lee HK, Takamiya K, Han JS, Man H, Kim CH, Rumbaugh G et al (2003). Phosphorylation of the AMPA receptor GluR1 subunit is required for synaptic plasticity and retention of spatial memory. Cell 112: 631-643.

Li N, Lee B, Liu RJ, Banasr M, Dwyer JM, Iwata M et al (2010). mTOR-dependent synapse formation underlies the rapid antidepressant effects of NMDA antagonists. Science 329: 959-964.

Lukoyanov NV, Paula-Barbosa MM (2000). A single high dose of dizocilpine produces long-lasting impairment of the water maze performance in adult rats. Neurosci Lett 285: 139-142.

Lynch G (2006). Glutamate-based therapeutic approaches: ampakines. Curr Opin Pharmacol 6: 82-88.

Mammen AL, Kameyama K, Roche KW, Huganir RL (1997). Phosphorylation of the alpha-amino-3-hydroxy-5-methylisoxazole4-propionic acid receptor GluR1 subunit by calcium/calmodulin-dependent kinase II. J Biol Chem 272: 32528-32533.

Manahan-Vaughan D, von Haebler D, Winter C, Juckel G, Heinemann U (2008). A single application of MK801 causes symptoms of acute psychosis, deficits in spatial memory, and impairment of synaptic plasticity in rats. Hippocampus 18: 125-134.

Minichiello L (2009). TrkB signalling pathways in LTP and learning. Nat Rev Neurosci 10: 850-860.

Muller WE, Mutschler E, Riederer P (1995). Noncompetitive NMDA receptor antagonists with fast open-channel blocking kinetics and strong voltage-dependency as potential therapeutic agents for Alzheimer's dementia. Pharmacopsychiatry 28: 113-124.

Normann C, Clark K (2005). Selective modulation of Ca(2+) influx pathways by 5 -HT regulates synaptic long-term plasticity in the hippocampus. Brain Res 1037: 187-193.

Palop JJ, Mucke L (2010). Amyloid-beta-induced neuronal dysfunction in Alzheimer's disease: from synapses toward neural networks. Nat Neurosci 13: 812-818.

Paoletti P, Bellone C, Zhou Q (2013). NMDA receptor subunit diversity: impact on receptor properties, synaptic plasticity and disease. Nat Rev Neurosci 14: 383-400.

Parsons CG, Stoffler A, Danysz W (2007). Memantine: a NMDA receptor antagonist that improves memory by restoration of homeostasis in the glutamatergic system-too little activation is bad, too much is even worse. Neuropharmacology 53: 699-723.

Rebola N, Srikumar BN, Mulle C (2010). Activity-dependent synaptic plasticity of NMDA receptors. J Physiol 588: 93-99.

Riedel G, Platt B, Micheau J (2003). Glutamate receptor function in learning and memory. Behav Brain Res 140: 1-47.

Sheng M, Sabatini BL, Sudhof TC (2012). Synapses and Alzheimer's disease. Cold Spring Harb Perspect Biol 4: a005777.

Shepherd JD, Huganir RL (2007). The cell biology of synaptic plasticity: AMPA receptor trafficking. Annu Rev Cell Dev Biol 23: 613-643. 
Talantova M, Sanz-Blasco S, Zhang X, Xia P, Akhtar MW, Okamoto S et al (2013). Abeta induces astrocytic glutamate release, extrasynaptic NMDA receptor activation, and synaptic loss. Proc Natl Acad Sci USA 110: E2518-E2527.

Tully T, Bourtchouladze R, Scott R, Tallman J (2003). Targeting the CREB pathway for memory enhancers. Nat Rev Drug Discov 2: 267-277.

Waltereit R, Weller M (2003). Signaling from cAMP/PKA to MAPK and synaptic plasticity. Mol Neurobiol 27: 99-106.

Waxman EA, Lynch DR (2005). N-methyl-D-aspartate receptor subtypes: multiple roles in excitotoxicity and neurological disease. Neuroscientist 11: 37-49.

Yamada KA (2000). Therapeutic potential of positive AMPA receptor modulators in the treatment of neurological disease. Expert Opin Investig Drugs 9: 765-778.

Ye WC, Ji NN, Zhao SX, Liu JH, Ye T, McKervey MA et al (1996). Triterpenoids from Pulsatilla chinensis. Phytochemistry 42: 799-802.

Yokoi N, Fukata M, Fukata Y (2012). Synaptic plasticity regulated by protein-protein interactions and posttranslational modifications. Int Rev Cell Mol Biol 297: 1-43.
Zhang DM, Lin SM, Lau CW, Yiu A, Wang J, Li Y et al (2010). Anemoside A3-induced relaxation in rat renal arteries: role of endothelium and $\mathrm{Ca} 2+$ channel inhibition. Planta Med 76: 1814-1819.

Zheng Y, Zhou F, Wu X, Wen X, Li Y, Yan B et al (2010). 23-Hydroxybetulinic acid from Pulsatilla chinensis (Bunge) Regel synergizes the antitumor activities of doxorubicin in vitro and in vivo. J Ethnopharmacol 128: 615-622.

(i) (2) This work is licensed under a Creative Commons Attribution-NonCommercial-ShareAlike $\quad 4.0$ International License. The images or other third party material in this article are included in the article's Creative Commons license, unless indicated otherwise in the credit line; if the material is not included under the Creative Commons license, users will need to obtain permission from the license holder to reproduce the material. To view a copy of this license, visit http://creativecommons.org/licenses/by-nc-sa/4.0/

Supplementary Information accompanies the paper on the Neuropsychopharmacology website (http://www.nature.com/npp) 